OPEN ACCESS

Edited by:

Lei Deng,

Central South University, China

Reviewed by:

Chen Qingfeng,

Guangxi University, China

Shihua Zhang,

Wuhan University of Science and

Technology, China

*Correspondence:

Zhiyi Zhang

zhangzhiyi2014@163.com

Specialty section:

This article was submitted to

Statistical Genetics and Methodology,

a section of the journal

Frontiers in Genetics

Received: 23 September 2021

Accepted: 20 October 2021

Published: 04 November 2021

Citation:

Zhang $H$, Wang $Y$, Feng J, Wang S,

Wang $Y$, Kong $W$ and Zhang $Z$ (2021)

Integrative Analysis for Elucidating

Transcriptomics Landscapes of

Systemic Lupus Erythematosus.

Front. Genet. 12:782005.

doi: 10.3389/fgene.2021.782005

\section{Integrative Analysis for Elucidating Transcriptomics Landscapes of Systemic Lupus Erythematosus}

\author{
Haihong Zhang ${ }^{1}$, Yanli Wang ${ }^{1}$, Jinghui Feng ${ }^{2}$, Shuya Wang ${ }^{1}$, Yan Wang ${ }^{1}$, Weisi Kong ${ }^{1}$ and \\ Zhiyi Zhang ${ }^{1 *}$
}

${ }^{1}$ Department of Rheumatology and Immunology, The First Affiliated Hospital of Harbin Medical University, Harbin, China, ${ }^{2}$ Department of Gerontology, The First Affiliated Hospital of Harbin Medical University, Harbin, China

Systemic lupus erythematosus (SLE) is a complex and heterogeneous autoimmune disease that the immune system attacks healthy cells and tissues. SLE is difficult to get a correct and timely diagnosis, which makes its morbidity and mortality rate very high. The pathogenesis of SLE remains to be elucidated. To clarify the potential pathogenic mechanism of SLE, we performed an integrated analysis of two RNA-seq datasets of SLE. Differential expression analysis revealed that there were 4,713 and 2,473 differentially expressed genes, respectively, most of which were up-regulated. After integrating differentially expressed genes, we identified 790 common differentially expressed genes (DEGs). Gene functional enrichment analysis was performed and found that common differentially expressed genes were significantly enriched in some important immune-related biological processes and pathways. Our analysis provides new insights into a better understanding of the pathogenic mechanisms and potential candidate markers for systemic lupus erythematosus.

Keywords: systemic lupus erythematosus, differential expression analysis, gene functional enrichment analysis, RNA-seq, protein-protein interaction

\section{INTRODUCTION}

Systemic lupus erythematosus is a chronic autoimmune disease (Beccastrini et al., 2013; Davies et al., 2021). Its clinical manifestations are heterogeneous and involve one or more organs such as skin, kidney, joints, and nervous system (Von Feldt, 1995; Adinolfi et al., 2016; Ronco et al., 2021). The latest data from the US Lupus Registry and published studies around the world can more accurately estimate the incidence and prevalence of SLE. It is estimated that the incidence of 23.2 cases per 100,000 people in North America is the highest in the world (Tsokos, 2011; Rees et al., 2017). SLE is a heterogeneous rheumatic systemic disease with extremely diverse clinical manifestations and diverse pathogenesis (Wu et al., 2021). In addition, it is one of the most varied diseases in its epidemiology and etiology, with different types of immune dysfunction (Oku and Atsumi, 2018). SLE patients' immune system activation is characterized by exaggerated B cells and T cells responses (Tsokos, 2011). The health-related quality of life of SLE patients is significantly impaired (Di Battista et al., 2018). To obtain a better diagnosis and treatment method, it is necessary to explore the pathogenesis of SLE.

Since the successful application of high-throughput technology, it has been widely used in almost all biological research fields (Hess et al., 2020). With the development of high-throughput technology (Hess et al., 2020), biological research has been transformed from a single gene level to a full 
transcriptome level, which has greatly advanced many research fields in biology (Wang et al., 2009; McDermaid et al., 2019). Cheng. et al. based on the genome-wide expression data of peripheral blood mononuclear cells (PBMC) of SLE patients found a novel marker of SLE (Cheng et al., 2021). Jiang. et al. discovered a new type of lncRNA that plays an important role in the pathogenesis of SLE based on the whole transcriptome data of PBMC of SLE patients (Jiang et al., 2021). However, these studies were only conducted on a single dataset, and there was heterogeneity between different datasets. Therefore, through a comprehensive analysis of multiple datasets, more robust results will be obtained.

In this study, we conducted a systematic analysis of two gene expression datasets of SLE. First, differential expression analysis was performed to obtain differentially expressed genes (DEGs) in each dataset. To obtain robust results, we intersected the DEGs s of the two datasets. We found that 790 genes were differentially expressed in both datasets. Finally, gene function enrichment analysis showed that common DEGs were enriched in immunerelated biological pathways. Overall, our research provided new insight into the molecular mechanism of SLE.

\section{MATERIALS AND METHODS}

\section{Datasets}

"Systemic Lupus Erythematosus" and "RNA-seq" were used as the keywords for searching the GEO database. The gene expression datasets of PBMC from freshly isolated healthy controls and SLE patients were downloaded from the GEO database (GSE162828 and GSE169080), the platforms used were GPL24676, and GPL20795. GSE162828 included 10 samples of peripheral blood mononuclear cells and was divided into the SLE group (5 samples) and healthy controls group (5 samples). GSE169080 included seven samples of peripheral blood mononuclear cells and was divided into SLE group (4 samples) and healthy controls group (3 samples) (Clough and Barrett, 2016; Cheng et al., 2021; Jiang et al., 2021).

\section{Data Pre-processing and Identification of Differential Expressed Genes}

$\mathrm{R}$ package DESeq2 (1.26.0) was used for the analysis of the original datasets (Love et al., 2014). $|\log \mathrm{FC}|>1$ and p. adj $<0.05$ were defined as the cutoff values for further analysis of DEGs. Volcano and heatmap were constructed by R package ggplot2. Venn plot (http://bioinformatics.psb.ugent.be/ webtools/Venn/) was used to draw the intersection of two databases.

\section{Analyzing of DEGs on Protein-Protein Interaction Network}

Protein-protein interaction (PPI) network analysis helps to study the molecular mechanism of diseases from a systematic perspective and discover new drug targets (Wu et al., 2019). STRING (https://string-db.org/) is a database covering more than 5,000 organisms with known and predicted proteinprotein interactions, providing direct (physical) and indirect (functional) associations (Szklarczyk et al., 2017). We used String (https://string-db.org/) to generate biological networks for proteins, and the results were analyzed by Cytoscape (Shannon et al., 2003; Szklarczyk et al., 2017).

\section{Gene Functional Enrichment Analysis}

Gene Ontology (GO) is an ontology widely used in the field of bioinformatics, which covers three aspects of biology: biological process (BP), cellular component (CC), and molecular function (MF) (Thomas, 2017). Kyoto Encyclopedia of Genes and Genomes (KEGG) is a biological system advanced function and utility database based on molecular-level information from genome sequencing and other high-throughput experimental technologies (Kanehisa et al., 2017). In this study, R package clusterProfiler was used to perform GO functional annotation and KEGG pathway enrichment analysis for DEGs (Yu et al., 2012).

\section{RESULTS}

\section{Differentially Expressed Genes Between SLE Patients and Healthy Controls}

To obtain abnormally expressed genes in SLE patients, we separately analyzed the differential expression of two GEO datasets (GSE162828 and GSE169080). As shown in Figure 1A, there were 4,713 DEGs, including 2,717 upregulated and 1,996 down-regulated in the GSE162828 dataset. In the GSE169080 dataset, there were 2,473 DEGs, including 1,552 up-regulated and 921 down-regulated (Figure 1B). In both datasets, the number of up-regulated DEGs was more than the number of down-regulated DEGs (Figure 1C). In the GSE162828 dataset, the up-regulated DEGs accounted for $56.7 \%$ of all DEGs. At the same time, the up-regulated DEGs accounted for $62.8 \%$ of all DEGs in the GSE169080 dataset. The trends in the two datasets were roughly the same.

In addition, the heatmap showed that DEGs can group samples by sample type, namely SLE patients (SLE) and healthy controls (Norm) (Figures 1D,E). These genes were highly concordant within groups. The expression level of these genes between SLE patients and healthy controls exhibited a large difference in both databases.

\section{Identification of Common Differentially Expressed Genes by Integrated Analysis}

Due to the heterogeneity between different datasets, the analysis results of different datasets may have certain differences (Ying et al., 2020). The gene expression in different samples may be different (Bao et al., 2021). To avoid this problem, integrating multiple datasets and a large number of samples help obtain more 

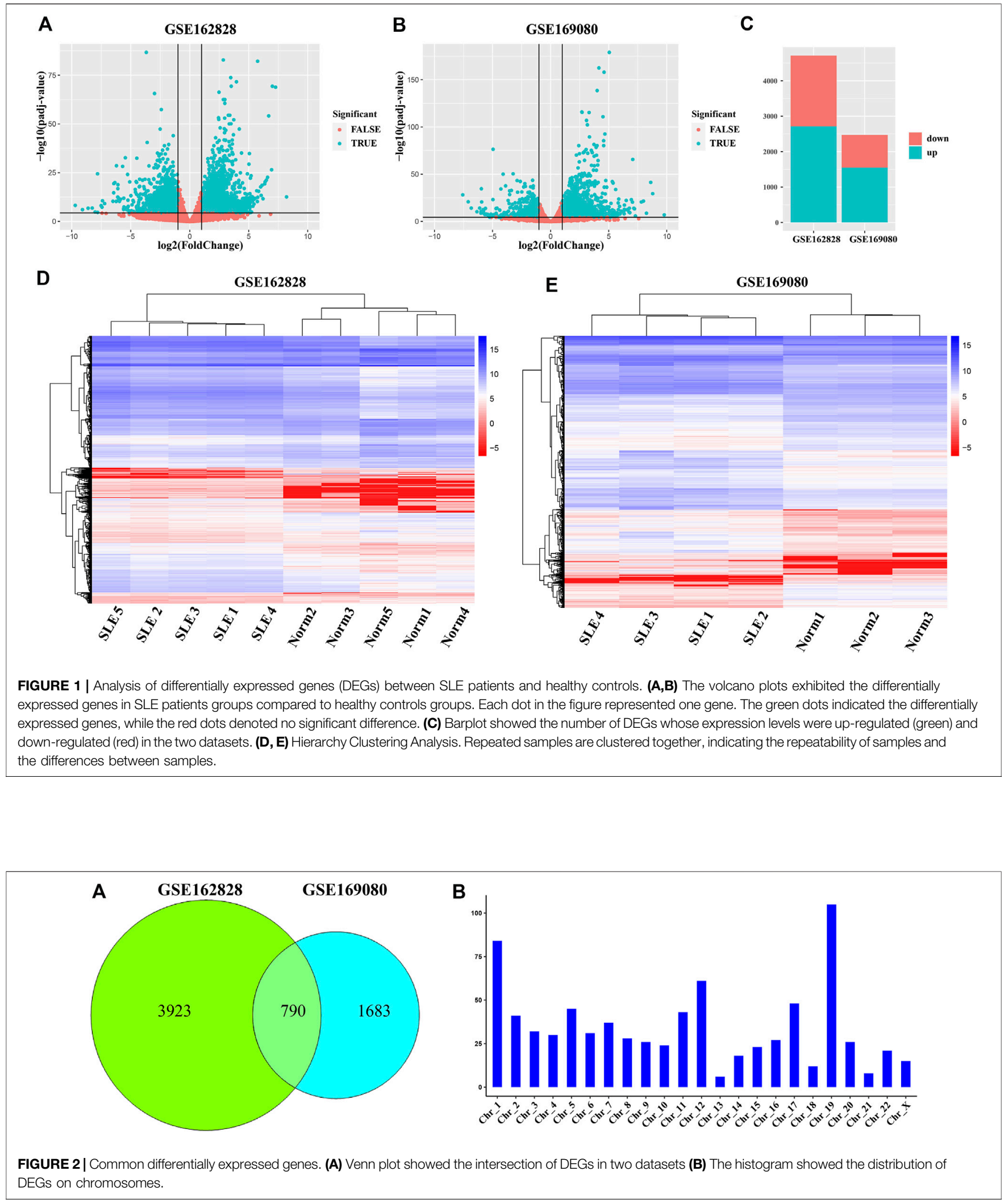


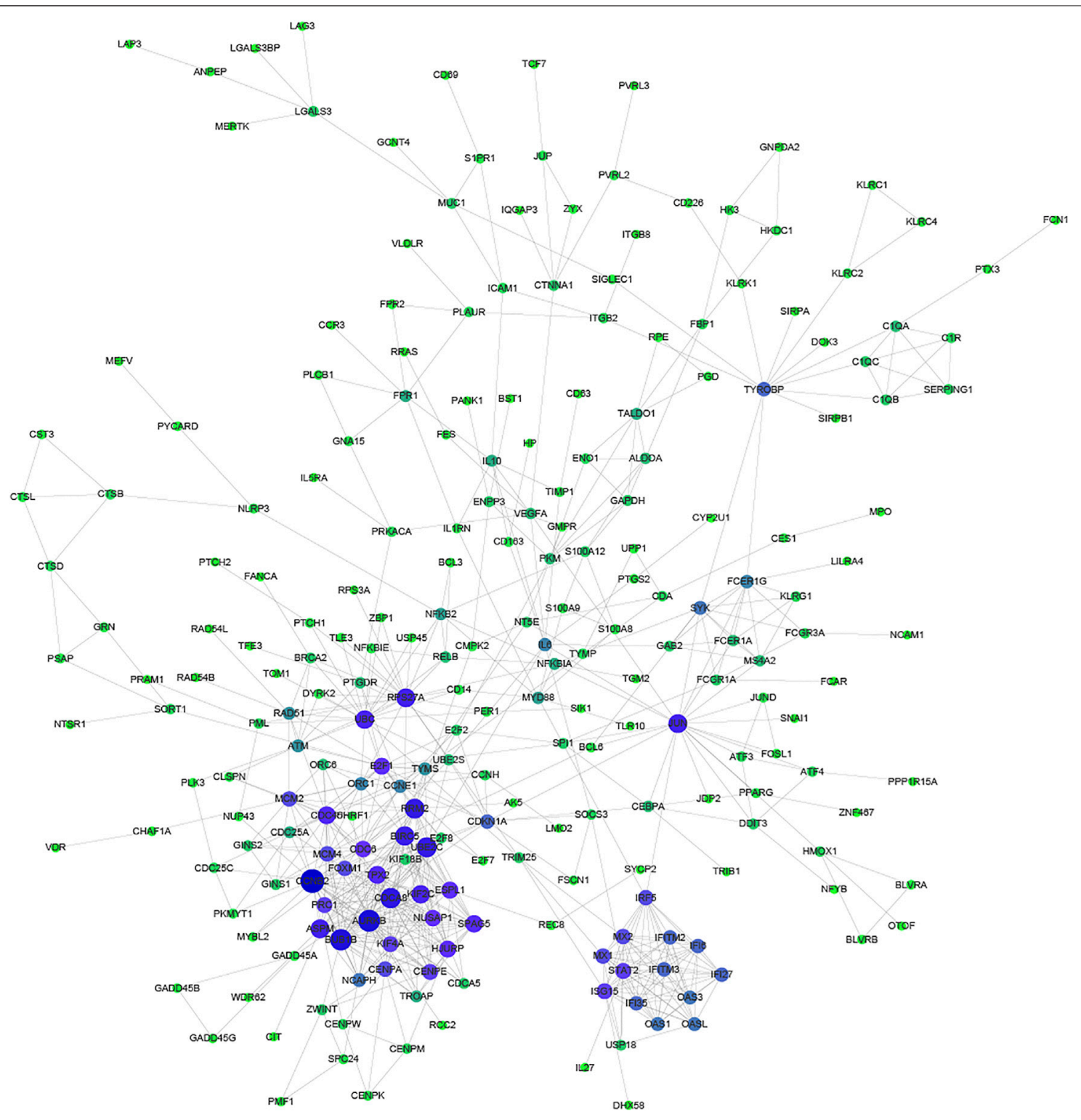

FIGURE 3 | Protein-Protein Interaction network of common DEGs. The size and color of the node depending on the degree, the larger the degree, the larger the node.

solid results (Kou et al., 2020). In this study, we integrated DEGs from two datasets to obtain common DEGs.

The Venn diagram showed that 790 DEGs were shared between the two datasets (Figure 2A). They accounted for $16.8 \%$ (GSE162828) and $31.9 \%$ (GSE169080) of the two datasets, respectively. There were 3,923 DEGs only in the GSE162828 dataset, and 1,683 DEGs only in GSE169080 dataset. This may be caused by different sequencing technologies and sample heterogeneity.

We defined these 790 DEGs as common DEGs. To further explore the distribution of common DEGs on the chromosomes, we had made statistics on the chromosomal locations of these genes. As shown in Figure 2B, we found that these genes were distributed on every chromosome. Most of these genes were distributed on chromosome 19. On the contrary, they were only 6 DEGs on chromosome 13.

\section{Analysis of Common Differentially Expressed Genes on Protein-Protein Interaction Network}

Proteins usually perform biological functions in concert. It has been shown that there is a close relationship between Protein-Protein Interaction (PPI) and the biological 


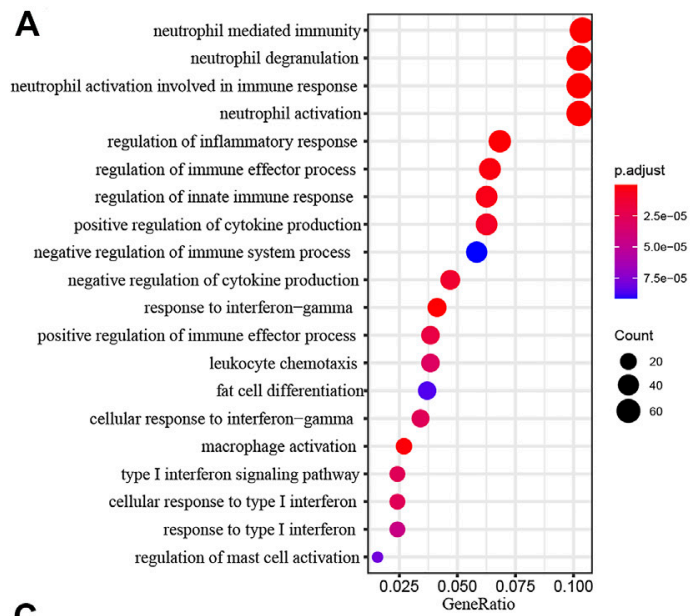

C

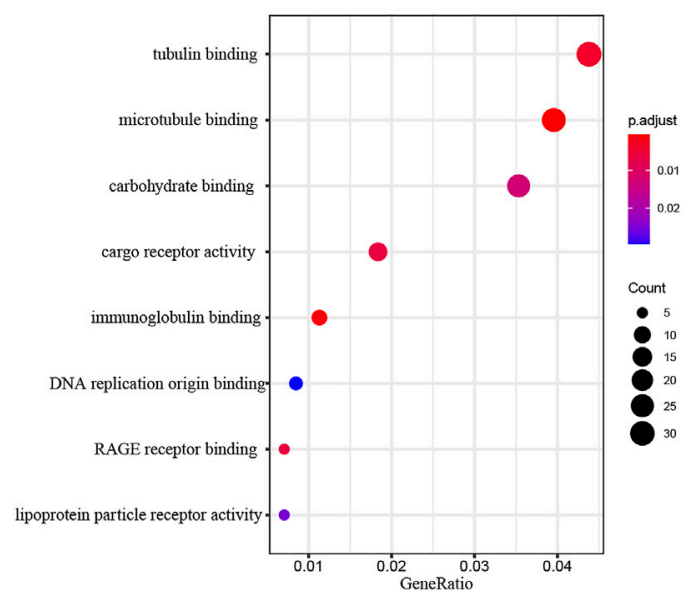

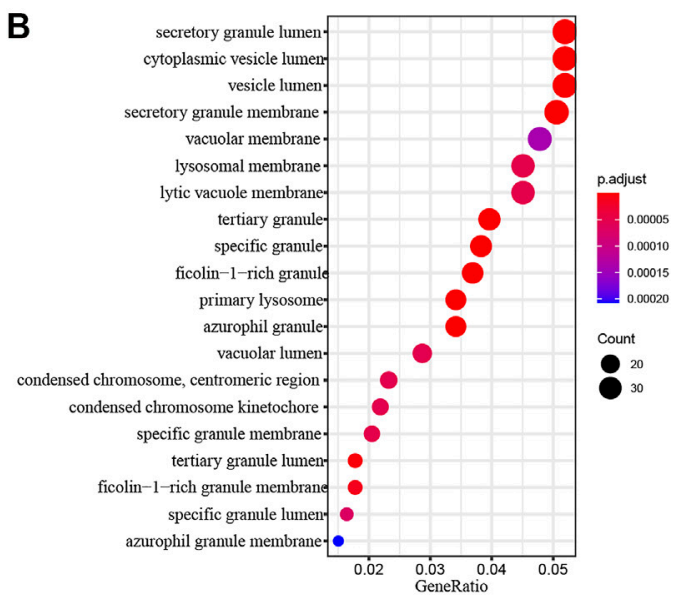

D

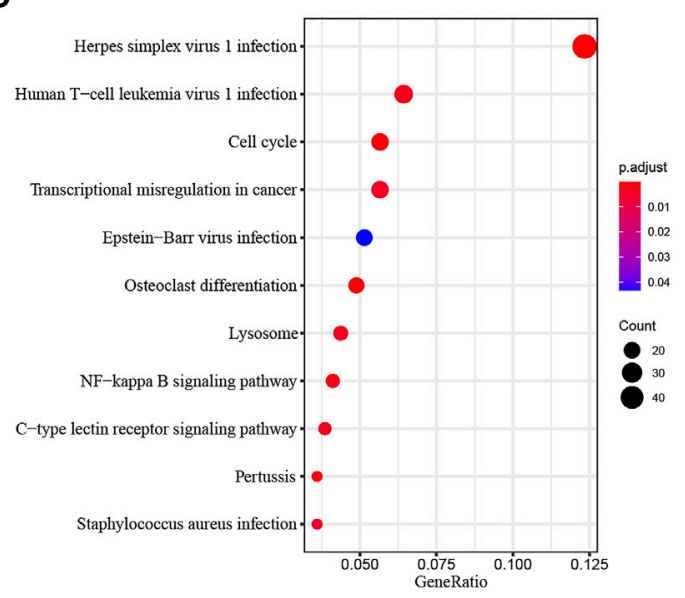

FIGURE 4 | Functional Enrichment Analysis of common DEGs. (A) Biological process analysis of common DEGs. (B) Cellular component analysis of common DEGs. (C) Molecular function analysis of common DEGs. (D) KEGG analysis of common DEGs.

functions of gene/protein clusters (Li H. et al., 2019). To further analyze the correlation between common DEGs, STRING and Cytoscape were used to construct the PPI network (Figure 3). Part of common DEGs was predicted to have a strong association with other genes. The size and color of the node depending on the degree, the larger the degree, the larger the node.

Especially, CCNB2, CDCA8, AURKB, BUB1B, RRM2, BIRC5, and $U B E 2 C$ had the largest degree. CCNB2 is an essential component of the cell cycle regulatory machinery (Takashima et al., 2014; Li R. et al., 2019). CDCA8 is an essential regulator of mitosis and cell division (Zhang et al., 2020). AURKB participates in the regulation of alignment and segregation of chromosomes during mitosis and meiosis through association with microtubules (Ahmed et al., 2021). BUB1B encodes a kinase involved in the spindle checkpoint function (Zhang et al., 2021). RRM2 encodes one of two non-identical subunits for ribonucleotide reductase (Mazzu et al., 2020). BIRC5 encodes negative regulatory proteins that prevent apoptotic cell death (Adamopoulos et al., 2021). UBE2C is required for the destruction of mitotic cyclins and cell cycle progression (Jin et al., 2020).

\section{Functional Enrichment Analysis of Common Differentially Expressed Genes}

To investigate the biological function of common DEGs, we used clusterProfiler to perform Functional enrichment analysis. Biological Process (BP) enrichment showed that the common DEGs were enriched in neutrophil mediated immunity, neutrophil degranulation, neutrophil activation involved in immune response, neutrophil activation and regulation of inflammatory response (Figure 4A). Cellular Component (CC) enrichment showed that the common DEGs were mainly enriched in secretory granule lumen, cytoplasmic vesicle lumen, vesicle lumen, secretory granule membrane and vacuolar membrane (Figure 4B). Molecular Function (MF) enrichment showed that the common DEGs were significantly 
enriched in tubulin binding, microtubule binding, carbohydrate binding, cargo receptor activity and immunoglobulin binding (Figure 4C).

KEGG pathway analysis provided a potential functional cluster of common DEGs, indicating that the common DEGs were clustered in Herpes simplex virus one infection, Human T-cell leukemia virus one infection, Cell cycle, Transcriptional misregulation in cancer and Epstein-Barr virus infection (Figure 4D).

\section{DISCUSSION}

SLE is a multi-system autoimmune inflammation that can affect multiple organs and cause extensive and severe clinical manifestations (Wu et al., 2021). The current understanding of the pathogenesis of SLE is not comprehensive. The key driving factors involved in the occurrence and development of SLE remain to be determined. In this study, we provided new insights into the transcriptome of SLE based on RNAseq data.

The results showed that compared with the normal healthy control groups, a large number of genes in SLE patients were abnormally expressed. Through integrated analysis, we found that there were 790 shared DEGs in the two databases. The results indicated that these common DEGs may lead to the occurrence and development of SLE. Previous studies had shown that lncRNA and circRNA are important factors leading to the occurrence of SLE (Cheng et al., 2021; Jiang et al., 2021). We found that the differential expression of these common DEGs might play an important role in this process.

\section{REFERENCES}

Adamopoulos, P. G., Tsiakanikas, P., Adam, E. E., and Scorilas, A. (2021). Unraveling Novel Survivin mRNA Transcripts in Cancer Cells Using an InHouse Developed Targeted High-Throughput Sequencing Approach. Genomics 113 (1 Pt 2), 573-581. doi:10.1016/j.ygeno.2020.09.053

Adinolfi, A., Valentini, E., Calabresi, E., Tesei, G., Signorini, V., Barsotti, S., et al. (2016). One Year in Review 2016: Systemic Lupus Erythematosus. Clin. Exp. Rheumatol. 34 (4), 569-574.

Ahmed, A., Shamsi, A., Mohammad, T., Hasan, G. M., Islam, A., and Hassan, M. I. (2021). Aurora B Kinase: a Potential Drug Target for Cancer Therapy. J. Cancer Res. Clin. Oncol. 147 (8), 2187-2198. doi:10.1007/ s00432-021-03669-5

Bao, X., Shi, R., Zhao, T., Wang, Y., Anastasov, N., Rosemann, M., et al. (2021). Integrated Analysis of Single-Cell RNA-Seq and Bulk RNA-Seq Unravels Tumour Heterogeneity Plus M2-like Tumour-Associated Macrophage Infiltration and Aggressiveness in TNBC. Cancer Immunol. Immunother. 70 (1), 189-202. doi:10.1007/s00262-020-02669-7

Beccastrini, E., D'Elios, M. M., Emmi, G., Silvestri, E., Squatrito, D., Prisco, D., et al. (2013). Systemic Lupus Erythematosus: Immunopathogenesis and Novel Therapeutic Targets. Int. J. Immunopathol Pharmacol. 26 (3), 585-596. doi:10.1177/039463201302600302

Cheng, Q., Chen, M., Chen, X., Chen, X., Jiang, H., Wu, H., et al. (2021). Novel Long Non-coding RNA Expression Profile of Peripheral Blood Mononuclear Cells Reveals Potential Biomarkers and Regulatory Mechanisms in Systemic Lupus Erythematosus. Front. Cel Dev. Biol. 9, 639321. doi:10.3389/ fcell.2021.639321

Clough, E., and Barrett, T. (2016). The Gene Expression Omnibus Database. Methods Mol. Biol. 1418, 93-110. doi:10.1007/978-1-4939-3578-9_5
Through further analysis, we found that the DEGs tended to up-regulated in the two datasets. Through protein-protein interaction network analysis of commonly dysregulated genes, we found that there was a strong correlation between these genes. These PPI networks may have affected the occurrence and development of SLE. Pathway enrichment results showed that common DEGs were significantly enriched in immune-related pathways such as neutrophil mediated immunity, neutrophil degranulation, neutrophil activation involved in the immune response.

In summary, we integrated and analyzed high-throughput sequencing RNA-seq datasets to uncover potential molecular mechanisms of SLE. Our findings provide new clues for possible targeted therapy of SLE. Further studies on the functions of those common DEGs hoped to better understand SLE by integrating more data.

\section{DATA AVAILABILITY STATEMENT}

Publicly available datasets were analyzed in this study. This data can be found here: https://www.ncbi.nlm.nih.gov/geo/ GSE162828 and GSE169080

\section{AUTHOR CONTRIBUTIONS}

$\mathrm{ZZ}$ designed the experiments. HZ obtained the data from GEO. YW, JF, SW, YW, and WK analyzed the data. HZ and ZZ wrote the manuscript. All authors read and approved the manuscript.

Davies, K., Dures, E., and Ng, W.-F. (2021). Fatigue in Inflammatory Rheumatic Diseases: Current Knowledge and Areas for Future Research. Nat. Rev. Rheumatol. 17, 651-664. doi:10.1038/s41584-021-00692-1

Di Battista, M., Marcucci, E., Elefante, E., Tripoli, A., Governato, G., Zucchi, D., et al. (2018). One Year in Review 2018: Systemic Lupus Erythematosus. Clin. Exp. Rheumatol. 36 (5), 763-777.

Hess, J. F., Kohl, T. A., Kotrová, M., Rönsch, K., Paprotka, T., Mohr, V., et al. (2020). Library Preparation for Next Generation Sequencing: A Review of Automation Strategies. Biotechnol. Adv. 41, 107537. doi:10.1016/ j.biotechadv.2020.107537

Jiang, Z., Zhong, Z., Miao, Q., Zhang, Y., Ni, B., Zhang, M., et al. (2021). circPTPN22 as a Novel Biomarker and ceRNA in Peripheral Blood Mononuclear Cells of Rheumatoid Arthritis. Mol. Med. Rep. 24 (2), 617. doi:10.3892/mmr.2021.12256

Jin, Z., Zhao, X., Cui, L., Xu, X., Zhao, Y., Younai, F., et al. (2020). UBE2C Promotes the Progression of Head and Neck Squamous Cell Carcinoma. Biochem. Biophysical Res. Commun. 523 (2), 389-397. doi:10.1016/j.bbrc.2019.12.064

Kanehisa, M., Furumichi, M., Tanabe, M., Sato, Y., and Morishima, K. (2017). KEGG: New Perspectives on Genomes, Pathways, Diseases and Drugs. Nucleic Acids Res. 45 (D1), D353-D361. doi:10.1093/nar/gkw1092

Kou, N., Zhou, W., He, Y., Ying, X., Chai, S., Fei, T., et al. (2020). A Mendelian Randomization Analysis to Expose the Causal Effect of IL-18 on Osteoporosis Based on Genome-wide Association Study Data. Front. Bioeng. Biotechnol. 8, 201. doi:10.3389/fbioe.2020.00201

Li, H., Long, J., Xie, F., Kang, K., Shi, Y., Xu, W., et al. (2019a). Transcriptomic Analysis and Identification of Prognostic Biomarkers in Cholangiocarcinoma. Oncol. Rep. 42 (5), 1833-1842. doi:10.3892/or.2019.7318

Li, R., Jiang, X., Zhang, Y., Wang, S., Chen, X., Yu, X., et al. (2019b). Cyclin B2 Overexpression in Human Hepatocellular Carcinoma Is Associated with Poor Prognosis. Arch. Med. Res. 50 (1), 10-17. doi:10.1016/j.arcmed.2019.03.003 
Love, M. I., Huber, W., and Anders, S. (2014). Moderated Estimation of Fold Change and Dispersion for RNA-Seq Data with DESeq2. Genome Biol. 15 (12), 550. doi:10.1186/s13059-014-0550-8

Mazzu, Y. Z., Armenia, J., Nandakumar, S., Chakraborty, G., Yoshikawa, Y., Jehane, L. E., et al. (2020). Ribonucleotide Reductase Small Subunit M2 Is a Master Driver of Aggressive Prostate Cancer. Mol. Oncol. 14 (8), 1881-1897. doi:10.1002/1878-0261.12706

McDermaid, A., Monier, B., Zhao, J., Liu, B., and Ma, Q. (2019). Interpretation of Differential Gene Expression Results of RNA-Seq Data: Review and Integration. Brief Bioinform 20 (6), 2044-2054. doi:10.1093/bib/bby067

Oku, K., and Atsumi, T. (2018). Systemic Lupus Erythematosus: Nothing Stale Her Infinite Variety. Mod. Rheumatol. 28 (5), 758-765. doi:10.1080/ 14397595.2018.1494239

Rees, F., Doherty, M., Grainge, M. J., Lanyon, P., and Zhang, W. (2017). The Worldwide Incidence and Prevalence of Systemic Lupus Erythematosus: a Systematic Review of Epidemiological Studies. Rheumatology (Oxford) 56 (11), 1945-1961. doi:10.1093/rheumatology/kex260

Ronco, P., Beck, L., Debiec, H., Fervenza, F. C., Hou, F. F., Jha, V., et al. (2021). Membranous Nephropathy. Nat. Rev. Dis. Primers 7 (1), 69. doi:10.1038/ s41572-021-00303-Z

Shannon, P., Markiel, A., Ozier, O., Baliga, N. S., Wang, J. T., Ramage, D., et al. (2003). Cytoscape: a Software Environment for Integrated Models of Biomolecular Interaction Networks. Genome Res. 13 (11), 2498-2504. doi:10.1101/gr.1239303

Szklarczyk, D., Morris, J. H., Cook, H., Kuhn, M., Wyder, S., Simonovic, M., et al. (2017). The STRING Database in 2017: Quality-Controlled Protein-Protein Association Networks, Made Broadly Accessible. Nucleic Acids Res. 45 (D1), D362-D368. doi:10.1093/nar/gkw937

Takashima, S., Saito, H., Takahashi, N., Imai, K., Kudo, S., Atari, M., et al. (2014). Strong Expression of Cyclin B2 mRNA Correlates with a Poor Prognosis in Patients with Non-small Cell Lung Cancer. Tumor Biol. 35 (5), 4257-4265. doi:10.1007/s13277-013-1556-7

Thomas, P. D. (2017). The Gene Ontology and the Meaning of Biological Function. Methods Mol. Biol. 1446, 15-24. doi:10.1007/978-1-4939-3743-1_2

Tsokos, G. C. (2011). Systemic Lupus Erythematosus. N. Engl. J. Med. 365 (22), 2110-2121. doi:10.1056/NEJMra1100359

Von Feldt, J. M. (1995). Systemic Lupus Erythematosus. Postgrad. Med. 97 (483), 7986-7994. doi:10.1080/00325481.1995.11945982

Wang, Z., Gerstein, M., and Snyder, M. (2009). RNA-seq: a Revolutionary Tool for Transcriptomics. Nat. Rev. Genet. 10 (1), 57-63. doi:10.1038/nrg2484
Wu, D., Ai, L., Sun, Y., Yang, B., Chen, S., Wang, Q., et al. (2021). Role of NLRP3 Inflammasome in Lupus Nephritis and Therapeutic Targeting by Phytochemicals. Front. Pharmacol. 12, 621300. doi:10.3389/fphar.2021.621300

Wu, H.-T., Chen, W.-T., Li, G.-W., Shen, J.-X., Ye, Q.-Q., Zhang, M.-L., et al. (2019). Analysis of the Differentially Expressed Genes Induced by Cisplatin Resistance in Oral Squamous Cell Carcinomas and Their Interaction. Front. Genet. 10, 1328. doi:10.3389/fgene.2019.01328

Ying, X., Jin, X., Wang, P., He, Y., Zhang, H., Ren, X., et al. (2020). Integrative Analysis for Elucidating Transcriptomics Landscapes of Glucocorticoid-Induced Osteoporosis. Front. Cel Dev. Biol. 8, 252. doi: $10.3389 /$ fcell.2020.00252

Yu, G., Wang, L.-G., Han, Y., and He, Q.-Y. (2012). clusterProfiler: an R Package for Comparing Biological Themes Among Gene Clusters. OMICS: A J. Integr. Biol. 16 (5), 284-287. doi:10.1089/omi.2011.0118

Zhang, C., Zhao, L., Leng, L., Zhou, Q., Zhang, S., Gong, F., et al. (2020). CDCA8 Regulates Meiotic Spindle Assembly and Chromosome Segregation during Human Oocyte Meiosis. Gene 741, 144495. doi:10.1016/j.gene.2020.144495

Zhang, P. C., Zhong, X. R., Zheng, H., Li, L., Chen, F., Shen, M. J., et al. (2021). Prognostic Values of Spindle Checkpoint Protein BUB1B in Triple Negative Breast Cancer. Zhonghua Bing Li Xue Za Zhi 50 (6), 645-649. doi:10.3760/ cma.j.cn112151-20210131-00108

Conflict of Interest: The authors declare that the research was conducted in the absence of any commercial or financial relationships that could be construed as a potential conflict of interest.

Publisher's Note: All claims expressed in this article are solely those of the authors and do not necessarily represent those of their affiliated organizations, or those of the publisher, the editors and the reviewers. Any product that may be evaluated in this article, or claim that may be made by its manufacturer, is not guaranteed or endorsed by the publisher.

Copyright (c) 2021 Zhang, Wang, Feng, Wang, Wang, Kong and Zhang. This is an open-access article distributed under the terms of the Creative Commons Attribution License (CC BY). The use, distribution or reproduction in other forums is permitted, provided the original author(s) and the copyright owner(s) are credited and that the original publication in this journal is cited, in accordance with accepted academic practice. No use, distribution or reproduction is permitted which does not comply with these terms. 